\title{
Diagnóstico de Factores de Deserción de un Programa Educativo
}

\section{Diagnosis of Desert Factors of an Educational Program}

\author{
Calderón-Rodríguez Marysol de Fátima ${ }^{1 *}$, Olea-Miranda Jaime¹, Ochoa-Vázquez Ivan¹ \\ 1 Departamento de Ingeniería Industrial. Universidad de Sonora. Blvd. Luis Encinas y Rosales S/N,Col. Centro Hermosillo, Sonora, México.
}

Autor para la correspondencia: Calderón-Rodríguez Marysol de Fátima. maryfacaro@gmail.com

\section{Resumen}

El estudio analiza los resultados de un cuestionario realizado a los alumnos desertores de un programa educativo de ingeniería industrial. Se utilizó un análisis de correlación para explicar los posibles factores que determinan la deserción en sus estudios universitarios, el análisis factorial arrojó cuatro factores; factor institucional, factor individual dividido en; motivación, vocación y dedicación, factor académico y factor ambiental dividido en; económico, social y geográfico. Los resultados se estudiaron bajo un análisis de correlación y análisis factorial. Según los resultados el factor institucional es el que afecta directamente al fenómeno de la deserción para la población bajo estudio. Así mismo, los factores individuales, académicos y ambientales, aunque no son determinísticos también presentan una estrecha relación con el fenómeno.

Palabras clave: Deserción, diagnóstico, ingeniería industrial.

\begin{abstract}
The study analyzes the results of a questionnaire made to the students who dropped out of an industrial engineering education program. Using a correlation analysis to explain the possible factors that determine dropout in their university studies. The factorial analysis showed four factors; institutional factors, individual factors divided into; motivation, vocation and dedication, academic factors and environmental factors divided into; economic, social, and geographical. The results were studied under a correlation analysis and factor analysis. According to the results, the institutional factors are those that directly affect the phenomenon of desertion for the population under study, by the way, the individual, academic and environmental factors, even though they are deterministic, also have a close relationship with the phenomenon.
\end{abstract}

Key words: Desertion, diagnosis, industrial engineering.

DOI 10.46588/invurnus.v16i1.42

Recibido 05/05/2021

Aceptado 20/05/2021

Publicado 22/05/2021 


\section{Introducción}

Para Tinto (1975) la deserción es el proceso de abandono voluntario o forzoso de la carrera en que se matricula un estudiante, por la influencia positiva o negativa de circunstancias internas o externas al alumno y para Himmel (2002) la deserción es el abandono prematuro de un programa de estudios antes de alcanzar el titulo o grado, y considera un tiempo suficientemente largo como para descartar la posibilidad de que el estudiante se reincorpore, en este sentido, es relevante mencionar que las universidades se desarrollaron a partir de modelos centrados en el desarrollo disciplinario, dirigido a una élite intelectual, académica o económica, y que en la actualidad, el enfoque que la sociedad le ha brindado a la adquisición del conocimiento ha propiciado la tendencia del aumento de la cobertura de la educación superior, es decir, la masificación del acceso a la educación superior, lo que implica una población estudiantil más heterogénea; más grande y más diversa, (Fernández, Martínez-Conde y Melipillan, 2009; González y Uribe 2005; Lemaitre, 2003).

Como lo afirma Fernández et al (2009) si las IES (Instituciones de Educación Superior) no cuentan con los mecanismos adecuados para manejar la diversidad estudiantil, puede presentarse como efecto inherente una disminución considerable en los estándares de calidad, graduando profesionales que no alcancen el perfil de egreso necesario, previamente definido por las partes interesadas de cada programa educativo. Por otra parte, optar por no disminuir los estándares de calidad requeridos para el perfil de egreso, puede exponer a las IES a altas tasas de deserción, lo que tampoco les proporciona prestigio académico a las Universidades (Himmel, 2003).

Según Vries, León, Romero y Hernández (2011) existen factores que ayudan a mitigar la deserción, como lo es: la flexibilidad curricular, la organización administrativa, la infraestructura y el ambiente académico y social que influyen sobre la permanencia en la IES, podemos reconocer que existe un elemento de integración entre el individuo y la institución. En el caso de América Latina, González (2006) hace hincapié en factores como falta de apoyo financiero para estudiantes e instituciones, altas tasas de desempleo juvenil, la insuficiencia de la preparación previa, ausencia de orientación vocacional, o la falta de preparación y actualización de los docentes, entre otros.

En el caso de México Zúñiga (2008) argumenta que resultan más importantes los factores como la situación económica, la irregularidad de los estudios, o la reprobación de materias. Mori (2012) estimó que los factores ambientales, académicos, personales y de socialización universitaria son los que mejor explican el fenómeno de la deserción (figura 1), éste cuenta con múltiples causas y aristas teóricas, por lo cual, es necesario contextualizar correctamente el estudio para no caer en la generalización de los factores que causan la deserción para el presente estudio.

Existe un vasto conocimiento sobre la importancia de una sociedad más capacitada para idear, organizar y plantear óptimas soluciones a los problemas que se suscitan día con día, es por ello que unos de los principales objetivos del presente estudio es analizar los elementos que presentan mayor influencia en la deserción estudiantil, a partir del análisis de los factores involucrados, con la finalidad de elaborar estrategias encaminadas a un amplio e incluyente panorama institucional, en otras palabras, IES capaces de incorporar y homogeneizar la diversidad de la población estudiantil, y plantear como objetivo final egresados con altos estándares de calidad, la reducción de los índices de deserción y cumplir con el tiempo previamente establecido para la culminación del plan de estudios. 


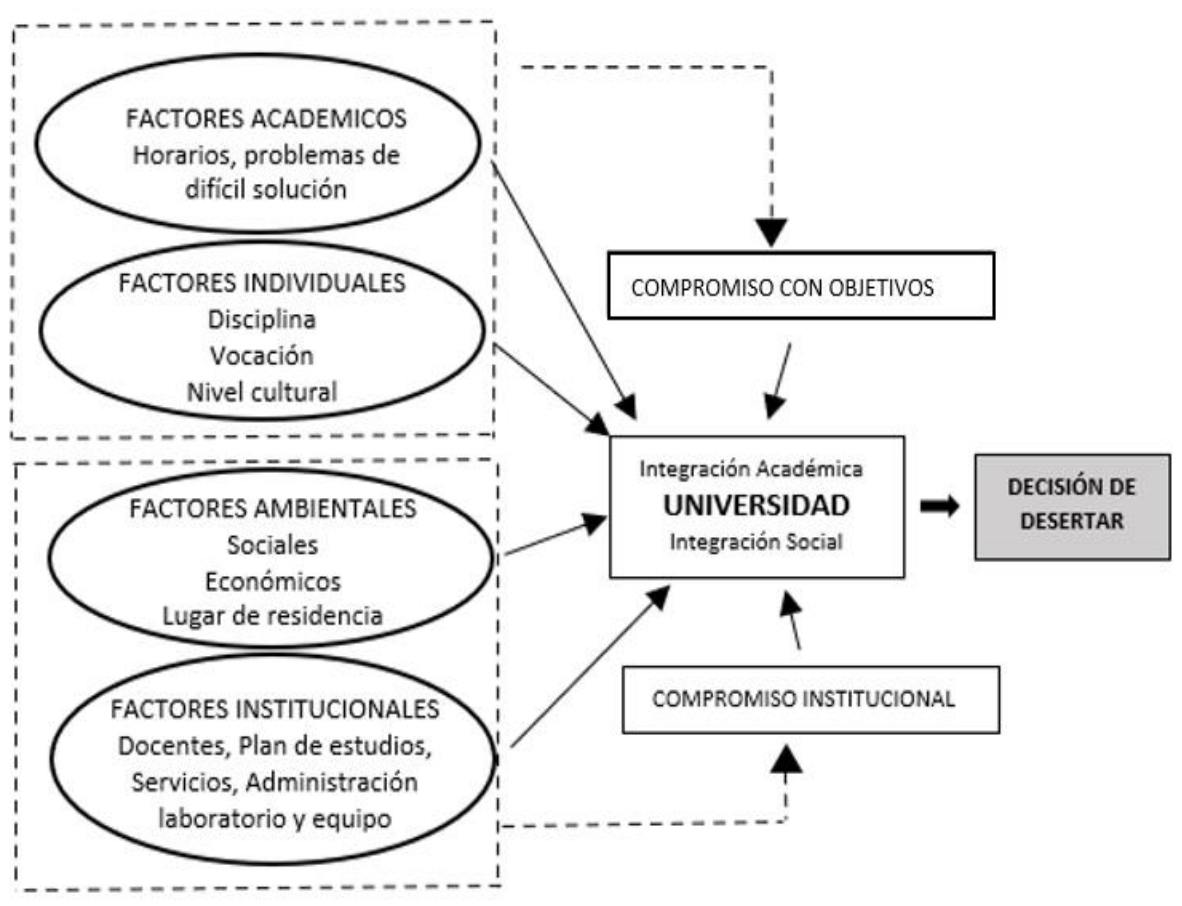

Figura 1. Modelo de análisis de deserción, Basado en Mori (2012)

\section{Metodología}

La investigación es del tipo no experimental, transversal y de correlación causal para el seguimiento de desertores en el programa educativo de Ingeniería Industrial para una Institución de Educación superior, se tomó la muestra de los ciclos académicos en el periodo semestral de 2014 a 2017, el cuestionario se aplicó en 2018 a personas pertenecientes a generaciones que entraron entre el 2014 y 2017, estas generaciones conforman un total de 216 alumnos aceptados como primer ingreso.

Basados en una población de 216 alumnos se identificaron 53 alumnos desertores, en promedio 13 alumnos por año, desertores equivalentes al $24.53 \%$ de este grupo, se encuestaron a 30 jóvenes lo que representa el $56.6 \%$ de los desertores identificados, la muestra tiene un margen de error de $10 \%$ y se consideró un intervalo de confianza del $90 \%$ para este análisis en particular.

El cuestionario utilizado fue desarrollado por Vries, León, Romero y Hernández en el estudio: ¿Desertores o Decepcionados? Distintas causas para abandonar los estudios universitarios en la Benemérita Universidad Autónoma de Puebla en el año 2010, el cuestionario que se aplicó contempla un total de 33 preguntas de las cuales 25 son de escala Likert y se refieren a las posibles causas para abandonar la Universidad y contempla factores académicos, factores individuales, ambientales e institucionales, las demás ocho preguntas sirven para caracterizar al alumno y su dedicación a la hora de realizar la encuesta. Los datos fueron obtenidos por llamada a teléfono celular, correo electrónico y entrevistas personales con el apoyo de estudiantes y excompañeros de los desertores de la Universidad. 
Al cuestionario se le aplicó el cálculo de coeficiente alfa de Cronbach, éste coeficiente obtiene las varianzas de las respuesta de los ítems que forman parte del instrumento y cuanto más se acerque a 1,0 mayor es la consistencia interna de los ítems analizados, en general, un valor considerado como aceptable para la confiabilidad del instrumento debe ser mayor a 0,70 (Cronbach, 1951) los cálculos de éste coeficiente se hicieron en el software Statistical Package for the Social Science (SPSS) versión 22, se obtuvo 0.769 del coeficiente de Cronbach, por lo tanto, podemos afirmar que el instrumento es adecuado para el análisis que estamos realizando. Una vez aplicado el cuestionario se realizaron las medias estadísticas de cada ítem y se realizó un diagrama de barras para analizar la información, además se realizó una matriz de correlación para determinar factores a partir de ítems específicos de Vries et al (2011) y finalmente se utilizó el análisis de factores para clasificar las principales causas de la deserción clasificadas como; académicos, individuales, ambientales e institucionales según Mori (2012).

\section{Resultados y Discusión}

Si bien es cierto que la mayoría de los estudiantes se ven en la necesidad de trabajar para costear sus estudios, eso es una condición normal, es decir, independientemente que sean desertores o no, el alumno se ve forzado a realizar actividades económicas para ser autosuficiente, económicamente hablando, en la figura 2 se muestra como la principal causa de deserción la necesidad de trabajar debido a la situación económica difícil como lo menciona Zuñiga (2008).

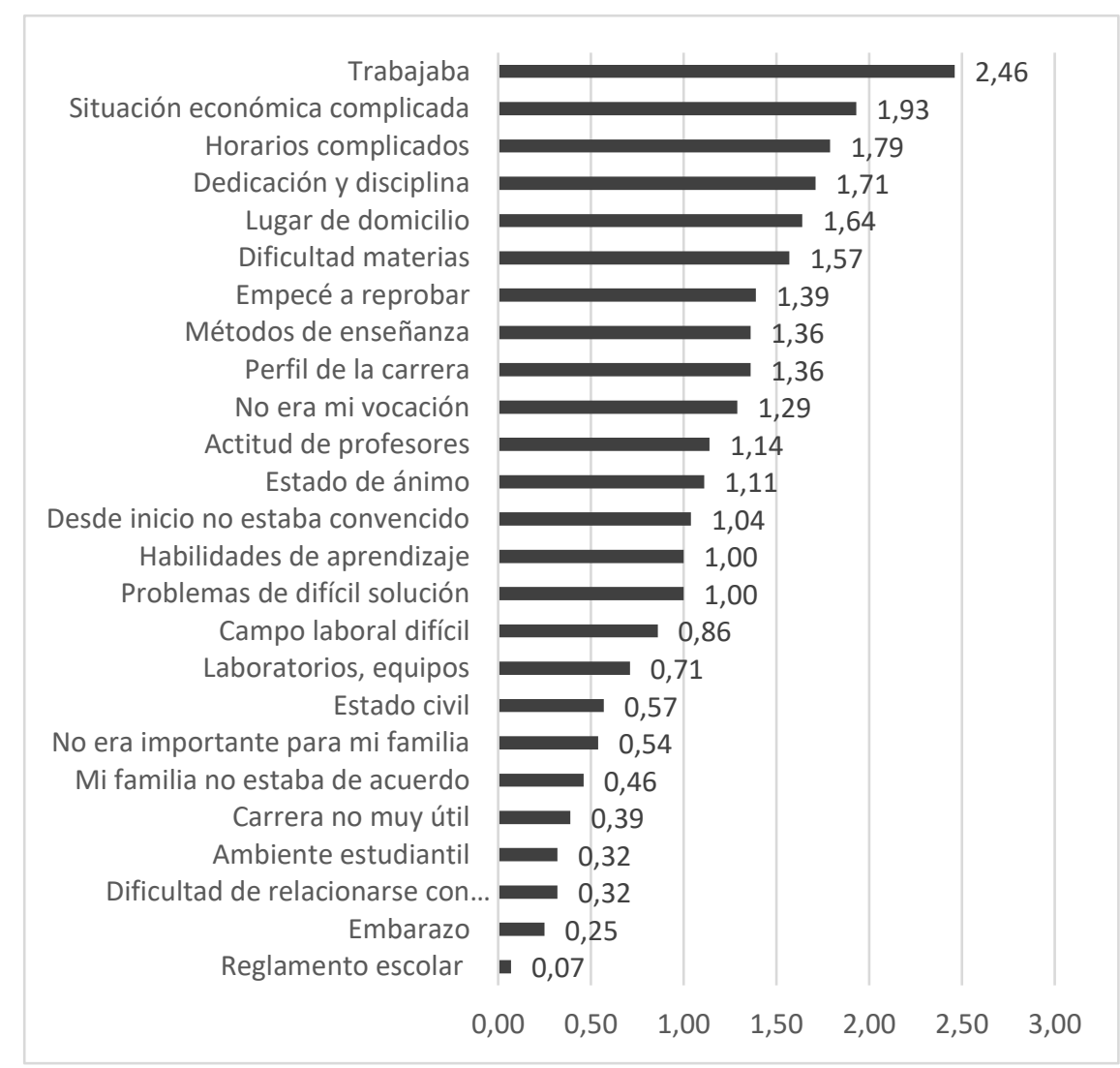

Figura 2. Razones para la deserción de los alumnos por orden de importancia (fuente: elaboración propia) 
De este modo, también es necesario precisar el hecho de que factores académicos como lo son, dedicación y disciplina, dificultad de materias y reprobación, son factores que tienen un peso importante en este análisis. En la tabla 1, se presenta la matriz de correlación simplificada, muestra como principal causa de deserción los factores institucionales, como lo son; actitud de los profesores y los métodos de enseñanza, con una correlación de Pearson de 0.795 y significancia del 0.000 , después con una correlación de 0.733 y significancia al 0.000 entre la actitud de los profesores y laboratorios y equipo.

Tabla 1. Matriz de correlación de las razones de la deserción.

\begin{tabular}{|c|c|c|c|c|}
\hline Factores de deserción & & $\begin{array}{l}\text { Perfil de } \\
\text { la carrera }\end{array}$ & $\begin{array}{c}\text { Métodos } \\
\text { de } \\
\text { enseñanza }\end{array}$ & $\begin{array}{l}\text { Actitud de } \\
\text { profesores }\end{array}$ \\
\hline \multirow{3}{*}{ Actitud de los profesores } & $\begin{array}{l}\text { Correlación } \\
\text { de Pearson }\end{array}$ & 0.351 & $0.795^{* *}$ & 1 \\
\hline & $\begin{array}{c}\text { Significancia } \\
\text { bilateral }\end{array}$ & 0.057 & 0.000 & \\
\hline & $\begin{array}{c}\text { Numero de } \\
\text { datos }\end{array}$ & 30 & 30 & 30 \\
\hline \multirow{3}{*}{ Estado de animo } & $\begin{array}{c}\text { Correlación } \\
\text { de Pearson }\end{array}$ & $0.721^{* *}$ & 0.127 & 0.094 \\
\hline & $\begin{array}{c}\text { Significancia } \\
\text { bilateral }\end{array}$ & 0.000 & 0.503 & 0.622 \\
\hline & $\begin{array}{c}\text { Numero de } \\
\text { datos }\end{array}$ & 30 & 30 & 30 \\
\hline \multirow{3}{*}{ Laboratorios, equipos } & $\begin{array}{c}\text { Correlación } \\
\text { de Pearson }\end{array}$ & $0.527^{* *}$ & $0.684^{* *}$ & $0.733^{* *}$ \\
\hline & $\begin{array}{c}\text { Significancia } \\
\text { bilateral }\end{array}$ & 0.003 & 0.000 & 0.000 \\
\hline & $\begin{array}{c}\text { Numero de } \\
\text { datos }\end{array}$ & 30 & 30 & 30 \\
\hline
\end{tabular}

*. La correlación es significativa en el nivel 0,05 (2 colas).

**. La correlación es significativa en el nivel 0,01 (2 colas).

Otra causa que es relevante mencionar es el factor individual, como lo es el estado de ánimo y el perfil de la carrera, con $\mathrm{r}$ de Pearson de 0.721 y significancia al 0.000 , en este sentido, se observa que el aspecto psicológico y social individual también poseen influencia, debido a que la actitud del docente también representa un peso significante para motivar en el estudio y terminar de convencer al estudiante lo importante que es la culminación de su carrera para la creación de riqueza en la región.

En la tabla 2 se presenta la prueba de confiabilidad los factores, resultaron tener valores propios (Eigenvectores) mayores a 1 y explican el $79.55 \%$ de la varianza. Los factores institucionales se dividen en dos sub-factores; docente y administradores, en cuanto al docente se observan las causas como su actitud, los métodos de enseñanza que utiliza y el nivel de dificultad que le impone a su materia. El otro factor de la administración engloba lo relacionado con aspectos de planeación y gestión, aspectos como lo son la 
infraestructura física, las políticas de la universidad y el perfil de la carrera en total los dos sub-factores representan un $23.43 \%$ de la varianza de los datos con respecto al fenómeno analizado.

Tabla 2. Varianza total explicada y determinación de los factores comunes.

\begin{tabular}{cccc}
\hline & \multicolumn{3}{c}{ Índice o respuesta } \\
\cline { 2 - 4 } $\begin{array}{c}\text { Factores } \\
\text { comunes }\end{array}$ & Total & $\begin{array}{c}\text { \% de la } \\
\text { varianza }\end{array}$ & $\begin{array}{c}\% \\
\text { acumulado }\end{array}$ \\
\hline $\begin{array}{c}\text { Factores } \\
\text { Institucionales } \\
\text { Factores }\end{array}$ & 6.081 & 24.326 & 24.326 \\
$\begin{array}{c}\text { motivacionales } \\
\text { Factores }\end{array}$ & 3.056 & 12.224 & 36.550 \\
$\begin{array}{c}\text { académicos } \\
\text { Factores }\end{array}$ & 2.906 & 11.624 & 48.174 \\
$\begin{array}{c}\text { económicos } \\
\text { Factores } \\
\text { geográficos } \\
\text { Factores } \\
\text { sociales }\end{array}$ & 1.940 & 7.760 & 64.602 \\
$\begin{array}{c}\text { Factores } \\
\text { vocacionales } \\
\text { Factores de } \\
\text { dedicación }\end{array}$ & 1.493 & 5.972 & 70.575 \\
\hline Método de extracción: análisis de componentes principales.
\end{tabular}

El otro factor individual motivacional tiene que ver con su desempeño estudiantil y aspectos de estado de ánimo se muestran con un $12.33 \%$ de aportación al fenómeno. Seguido del factor académico relacionado a horarios, aprendizaje y la dificultad de la carrera tiene un peso del $11.33 \%$. En la tabla 3 se especifica con mayor grado de detalle basado en los factores de Mori 2012 y el cuestionario de Vries et al 2011. Dentro de los factores ambientales se encuentra el económico, donde el alumno debe de trabajar para pagar sus estudios o ayudar a su familia, este aspecto representa el $8.5 \%$ y se relaciona al lugar de origen (geográficos), si el alumno es foráneo tiene una situación económica complicada debido al lugar de origen este se reporta con $7.5 \%$ de aportación a la varianza. El último factor ambiental es el social, aspecto de integración social y de pertenencia es $6.8 \%$. Por último, una vez más, se mencionan aspectos individuales como lo es la vocación con un $4.76 \%$ y la dedicación $4.4 \%$ de aportación al fenómeno de deserción. Véase tabla 3.

\section{Conclusiones}

Es importante promover soluciones tecnológicas, proporcionar programas de actualización disciplinar a los académicos de acuerdo con las necesidades específicas del departamento de ingeniería industrial, modelos de enseñanza más involucrados con la diversidad social y tecnológica en la que nos encontramos inmersos. 
Otro factor por mencionar es la vinculación con el sector productivo, para mejorar los requerimientos necesarios en cuanto a conocimientos, habilidades y actitudes, de este modo facilitar la inserción del estudiante a la vida laboral.

Tabla 3. Factores que influyen en la deserción estudiantil de programa educativo de Ingeniería Industrial.

\begin{tabular}{|c|c|c|c|c|c|}
\hline FACTOR & SUBFACTOR & $\begin{array}{c}\text { Razones de la } \\
\text { deserción académica }\end{array}$ & Total & $\begin{array}{c}\text { Porcentaje } \\
\text { de la } \\
\text { varianza }\end{array}$ & $\begin{array}{l}\text { Porcentaje } \\
\text { acumulado }\end{array}$ \\
\hline \multirow{2}{*}{$\begin{array}{c}\text { Factores } \\
\text { institucionales }\end{array}$} & Docente & $\begin{array}{l}\text { Métodos de } \\
\text { enseñanza } \\
\text { Actitud del profesor } \\
\text { Dificultad de } \\
\text { materias }\end{array}$ & \multirow{2}{*}{5.85} & \multirow{2}{*}{23.43} & \multirow{2}{*}{23.43} \\
\hline & $\begin{array}{l}\text { Organización } \\
\text { Administrativa }\end{array}$ & $\begin{array}{l}\text { Laboratorio y equipos } \\
\text { Campo laboral difícil } \\
\text { Reglamento escolar } \\
\text { Perfil de la carrera } \\
\text { Carrera no muy útil }\end{array}$ & & & \\
\hline $\begin{array}{c}\text { Factores } \\
\text { individuales }\end{array}$ & Motivación & $\begin{array}{l}\text { Empecé a reprobar } \\
\text { Estado de animo } \\
\text { Estado civil }\end{array}$ & 3.08 & 12.33 & 35.76 \\
\hline $\begin{array}{c}\text { Factores } \\
\text { Académicos }\end{array}$ & Adaptación & $\begin{array}{l}\text { Horarios complicados } \\
\text { Habilidades de } \\
\text { aprendizaje } \\
\text { Problemas de difícil } \\
\text { solución }\end{array}$ & 2.83 & 11.33 & 47.09 \\
\hline \multirow{3}{*}{$\begin{array}{c}\text { Factores } \\
\text { Ambientales }\end{array}$} & Económico & Trabajaba & 2.13 & 8.5 & 55.59 \\
\hline & $\begin{array}{l}\text { Lugar de } \\
\text { procedencia }\end{array}$ & $\begin{array}{l}\text { Situación económica } \\
\text { complicada } \\
\text { Lugar de domicilio } \\
\text { Mi familia no estaba } \\
\text { de acuerdo }\end{array}$ & 1.8 & 7.5 & 63.09 \\
\hline & Social & Ambiente estudiantil & 1.69 & 6.8 & 69.89 \\
\hline \multirow{2}{*}{$\begin{array}{c}\text { Factores } \\
\text { Individuales }\end{array}$} & Vocación & No era mi vocación & 1.19 & 4.76 & 74.65 \\
\hline & Dedicación & $\begin{array}{l}\text { Dedicación y } \\
\text { disciplina }\end{array}$ & 1.09 & 4.4 & 79.05 \\
\hline
\end{tabular}

El mundo globalizado cada vez es más exigente, es por ello que cada vez son más los requerimientos de las empresas para contratar personal capaz de desarrollar aptitudes necesarias, por lo tanto, si el alumno tiene pleno conocimiento y reconocimiento de lo que representa como parte inherente de las actividades del sector industrial, aunado a una estrecha relación con la triada universidad-alumno-sector privado, los índices de 
productividad se elevarían exponencialmente, es decir, probablemente se observará decrecer la tasa de deserción.

\section{Referencias}

Fernández, O.; Martínez-Conde, M.; Melipillan, R. (2009) Estrategias de Aprendizaje y Autoestima: su relación con la permanencia y deserción universitaria. Estudios pedagógicos, Vol. 35, no. 1, p. 27-45.

González, M. (2006). Absentismo y abandono escolar: una situación singular de la exclusión educativa. REICE: Revista Electrónica Iberoamericana sobre calidad, eficacia y cambio en educación, Red Iberoamericana de Investigación sobre Cambio y Eficiencia Escolar (RINACE), Vol. 4, no. 1, p. 1-15.

Himmel, E. (2002). Modelos de análisis de la deserción estudiantil en la educación superior. Revista Calidad de la Educación. Consejo Superior de Educación. Ministerio de Educación, No.17, p. 91-108.

Himmel, E. (2003). Evaluación de aprendizajes en la Educación Superior: Una reflexión necesaria. Pensamiento Educativo. Vol. 33, p. 199-211.

Tinto, V. (1975). Dropout From Higher Education: A Theoretical Synthesis of Recent Research, Journal of Higher Education. $\mathrm{N}^{\circ}$ 45: 89-125.

Vries, W.; Arenas, L.; Romero J.; Hernández, S. (2011). ¿Desertores o decepcionados? Distintas causas para abandonar los estudios universitarios. Revista de la educación superior, Vol. 60, No.160, 29-49.

Zúñiga, M. (2008). Deserción estudiantil en el nivel superior. Causas y soluciones, México, Trillas.

Cómo citar este artículo: Calderón-Rodríguez, M., Olea-Miranda, J., \&Ochoa-Vázquez, I. (2021). Diagnóstico de Factores de Deserción de un Programa Educativo. INVURNUS, 16 (1) 1-8. 